NEWS

\title{
China rushes through major funding system
}

Next month, China will start pouring money into two long-awaited 'megaprojects' in infectious disease and drug discovery. But although scientists welcome the funding bonanza, many criticize how it is being administered - especially because, after waiting more than two years for the announcement, scientists were given only a few weeks to apply.

In February 2006, the national medium- and long-term programme for science and technology development, which lays out plans up to 2020 , called for investments in biomedical sciences. After debate among the ministries of health, science and technology, and industry - along with the powerful National Development and Reform Commission - it was decided that the health ministry would take the lead. But the negotiations took so long that requests for grant applications did not go out until the middle of last month.

Funding levels are not yet set, but insiders say roughly 6 billion yuan (US\$880 million) will be allocated for drug discovery and 3 billion yuan for infectious disease research. "The funding is huge - an unprecedented amount," says Ray Yip, who works on infectious disease at the Bill \& Melinda Gates Foundation in Beijing. In addition to university and institute researchers, industrial researchers can apply if their company is majority Chinese-owned.
Projects that win funding will run between October 2008 and December 2010. It is still unclear, however, whether all of the funding earmarked for this first stage will be squeezed into the next two years or allowed to carry over to the next Five Year Plan.

The infectious-disease money will go to research on HIV/AIDS, hepatitis B, hepatitis C and tuberculosis. The drug-discovery money will target ten major diseases including cancer, cardiovascular disease, neurodegenerative diseases, diabetes and mental illness. The biggest chunks of cash, however, will go towards the establishment of Good Laboratory Practice platforms - in which scientists establish procedures to ensure the accuracy of safety data of laboratory materials - and Good Clinical Practice platforms, which ensure the rights and the safety of patients involved in clinical trials. "The number-one goal is to make similar regulations to those in the United States and elsewhere," says Wei He, an immunologist at the Chinese Academy of Medical Sciences in Beijing, who was pulled in to organize the review of the applications in August and hasn't had a day off since.

Yip says that the financial lure of the megaprojects might help remedy China's shortage of experienced biomedical researchers. "It will no doubt attract talented people," he says. "Those

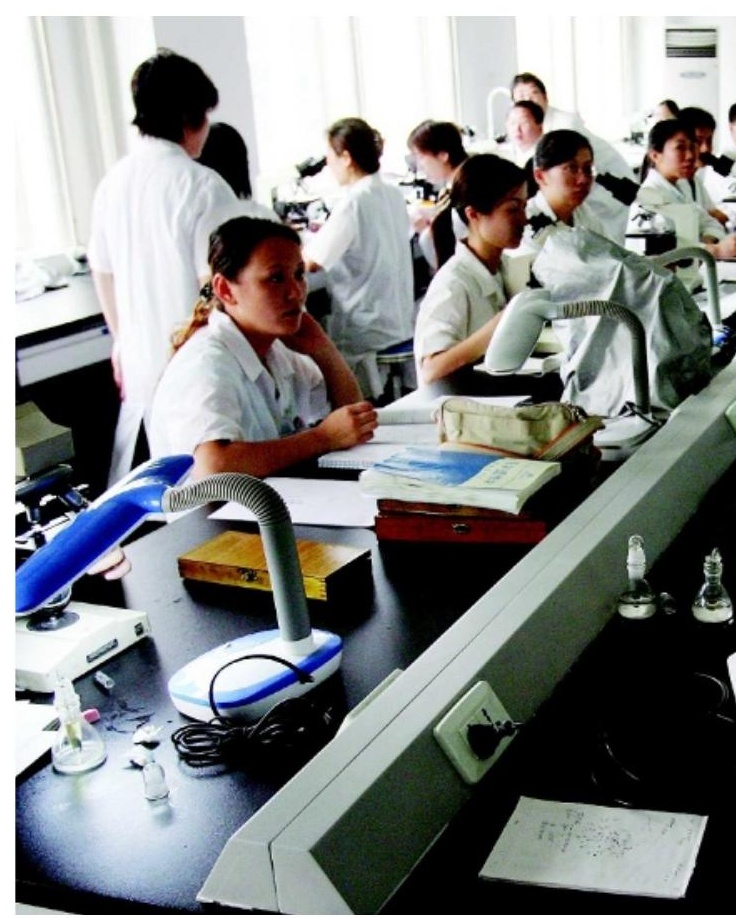

Reaction to China's new biomedical funding programmes has been mixed.

who didn't want to go back because the grants were too small will now find that they can get several million dollars to do a research project. The tables will start turning."

The projects are being rushed through in a post-Olympics scurry. The deadline for infectious disease applications was 31 August, just 16 days after the request for applications went up on the health ministry website. Likewise, researchers had only three weeks to meet the

\section{Megacity project seeks to gauge urban pollution}

Parisian pollution is nothing compared with that of Beijing or Mexico City. Yet Paris, with 11 million people crammed into a region just 20 kilometres across, is to take centre stage in a new research project on the impact of megacities on air pollution.

The MEGAPOLI project, which starts next month, will focus on building regional air-pollution models for every city in the world with a population of more than five million. It encompasses 23 research organizations from 11 European countries, along with 24 collaborating partners outside
Europe. Project scientists hope that it will lead to better maps of potential exposure to harmful aerosols and particles, and to improved urban planning.

Two dozen cities worldwide already have populations exceeding 10 million; much of the existing field data on what these do to the air come from studies of individual cities or regions. The novelty of

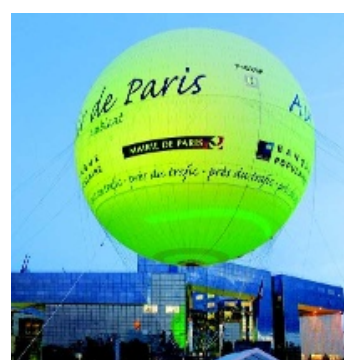

This colour of this balloon indicates Paris's air quality.
MEGAPOLI is to try to understand the bigger picture of the impact of megacities and the feedbacks between pollution and climate, says Alexander Baklanov of the Danish Meteorological Institute, a project leader. That includes, he says, studying atmospheric processes "from the street scale and up to the global scale, with interactions in both directions".
For all its grand goals, MEGAPOLI is budgeted at a relatively cheap $€ 3$ million (US\$4.3 million). That is because the project is $80 \%$ modelling and $20 \%$ measurements, says Mark Lawrence, a chemical transport modeller at the Max Planck Institute for Chemistry in Mainz, Germany. Much of the data have already been collected by its collaborating partners; MEGAPOLI will work, for instance, with the Milagro project, which carried out the largest air-pollution field campaign to date, in Mexico City in 2006.

MEGAPOLI will work with similar data sets from 11 other megacities, 
